\title{
Some applications of a hypergeometric identity
}

\author{
M. R. Eslahchi ${ }^{1} \cdot$ Mohammad Masjed-Jamei $^{2}$
}

Received: 6 July 2015 / Accepted: 7 November 2015/Published online: 25 November 2015

(c) The Author(s) 2015. This article is published with open access at Springerlink.com

\begin{abstract}
In this paper, we use a general identity for generalized hypergeometric series to obtain some new applications. The first application is a hypergeometric-type decomposition formula for elementary special functions and the second one is a generalization of the well-known Euler identity $e^{i x}=\cos x+i \sin x$ and an extension of hyperbolic functions in the sequel. Applying the mentioned identity on classical hypergeometric orthogonal polynomials and deriving summation formulae for some classical summation theorems are two further applications of this identity.
\end{abstract}

Keywords Hypergeometric functions - Euler identity · Hyperbolic functions - Classical hypergeometric orthogonal polynomials - Classical summation theorems

\section{Mathematical Subject Classification $33 \mathrm{C} 20 \cdot 33 \mathrm{C} 05$.} $33 \mathrm{C} 15$

\section{Introduction}

One of the main reasons for introducing and developing the generalized hypergeometric series is that many special functions $[2,15,16]$ can be represented in terms of them

M. R. Eslahchi

eslahchi@modares.ac.ir

Mohammad Masjed-Jamei

mmjamei@kntu.ac.ir; mmjamei@yahoo.com

1 Department of Applied Mathematics, Faculty of Mathematical Sciences, Tarbiat Modares University, P. O. Box 14115-134, Tehran, Iran

2 Department of Mathematics, K.N.Toosi University of Technology, P. O. Box 18315-1818, Tehran, Iran and therefore their initial properties can be directly found via the initial properties of hypergeometric functions. Also, they appear as solutions of many important ordinary differential equations $[7,11,20]$. Hence, finding any property of them may be valuable $[1,5,17]$. The generalized hypergeometric function

${ }_{p} F_{q}\left(\begin{array}{cccc}a_{1}, & a_{2}, \ldots & a_{p} \\ b_{1}, & b_{2}, & \ldots & b_{q}\end{array} \mid z\right)=\sum_{k=0}^{\infty} \frac{\left(a_{1}\right)_{k}\left(a_{2}\right)_{k} \ldots\left(a_{p}\right)_{k}}{\left(b_{1}\right)_{k}\left(b_{2}\right)_{k} \ldots\left(b_{q}\right)_{k}} \frac{z^{k}}{k !}$,

in which $(r)_{k}=\prod_{j=0}^{k-1}(r+j)=\Gamma(r+k) / \Gamma(r)$ denotes the Pochhammer symbol $[2,8]$ and $z$ may be a complex variable is indeed a Taylor series expansion for a function, say $f$, as $\sum_{k=0}^{\infty} c_{k}^{*} z^{k}$ with $c_{k}^{*}=f^{(k)}(0) / k$ ! for which the ratio of successive terms can be written as

$\frac{c_{k+1}^{*}}{c_{k}^{*}}=\frac{\left(k+a_{1}\right)\left(k+a_{2}\right) \ldots\left(k+a_{p}\right)}{\left(k+b_{1}\right)\left(k+b_{2}\right) \ldots\left(k+b_{q}\right)(k+1)}$.

According to the ratio test [6], the series (1) is convergent for any $p \leq q+1$. In fact, it converges in $|z|<1$ for $p=q+1$, converges everywhere for $p<q+1$ and converges nowhere $(z \neq 0)$ for $p>q+1$. Moreover, for $p=$ $q+1$ it absolutely converges on $|z|=1$ if the condition

$A^{*}=\operatorname{Re}\left(\sum_{j=1}^{q} b_{j}-\sum_{j=1}^{q+1} a_{j}\right)>0$,

holds and is conditionally convergent for $|z|=1$ and $z \neq 1$ if $-1<A^{*} \leq 0$ and is finally divergent for $|z|=1$ and $z \neq 1$ if $A^{*} \leq-1$.

This paper is organized as follows: in the next section, we use a general identity for any arbitrary hypergeometric series of type (1) to obtain some new applications. The first application is a hypergeometric-type decomposition 
formula for elementary special functions. The second application is a generalization of the well-known Euler identity $e^{i x}=\cos x+i \sin x$ and then an extension of hyperbolic functions. Applying the mentioned identity to classical hypergeometric orthogonal polynomials and deriving summation formulae for some classical summation theorems are two further applications of this identity.

\section{Some applications of a general identity for hypergeometric series}

The following result is given in [18, p. 441, formula 43] (see also [6]).

Corollary. For any natural number $m$ we have

$$
\begin{aligned}
& { }_{p} F_{q}\left(\begin{array}{ccc}
a_{1}, & a_{2}, \cdots & a_{p} \\
b_{1}, & b_{2}, \ldots & b_{q}
\end{array} \mid z\right) \\
& =\sum_{k=0}^{m-1} \frac{\left(a_{1}\right)_{k}\left(a_{2}\right)_{k} \cdots\left(a_{p}\right)_{k}}{\left(b_{1}\right)_{k}\left(b_{2}\right)_{k} \cdots\left(b_{q}\right)_{k}} \frac{z^{k}}{k !}{ }_{(m p+1)} F_{(m q+m)} \\
& \left.\left(\begin{array}{lll}
\vec{A}_{1, k}, & \vec{A}_{2, k}, \ldots & \vec{A}_{p, k}, 1 \\
\vec{B}_{1, k}, & \vec{B}_{2, k}, \ldots & \vec{B}_{q, k}, \vec{I}_{1, k}
\end{array}\right) m^{(p-q-1) m} z^{m}\right),
\end{aligned}
$$

where

$$
\begin{aligned}
\vec{A}_{j, k}= & \left(\frac{a_{j}+k}{m}, \frac{a_{j}+1+k}{m}, \ldots, \frac{a_{j}+m-1+k}{m}\right) \\
& (j=1,2, \ldots, p), \\
\vec{B}_{j, k}= & \left(\frac{b_{j}+k}{m}, \frac{b_{j}+1+k}{m}, \ldots, \frac{b_{j}+m-1+k}{m}\right) \\
& (j=1,2, \ldots, q),
\end{aligned}
$$

and

$\vec{I}_{1, k}=\left(\frac{1+k}{m}, \frac{2+k}{m}, \ldots, \frac{m+k}{m}\right)$.

Application 1. Identities (2) can be interpreted as a decomposition formula for many elementary special functions whose hypergeometric representations are known.

Example 1. Take $(p, q)=(0,0)$ and $m=2,3,4$ in (2) to, respectively, obtain

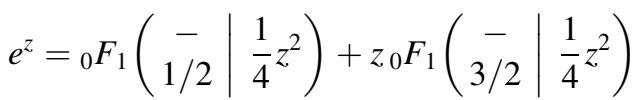

$$
\begin{aligned}
& =\cosh z+\sinh z \text {, }
\end{aligned}
$$

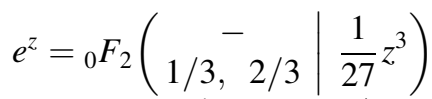

$$
\begin{aligned}
& +z_{0} F_{2}\left(\begin{array}{c|c}
- & - \\
2 / 3,4 / 3 & \frac{1}{27} z^{3}
\end{array}\right) \\
& +\frac{z^{2}}{2}{ }_{0} F_{2}\left(\begin{array}{c|c}
- & - \\
4 / 3,5 / 3 & \frac{1}{27} z^{3}
\end{array}\right) \text {, }
\end{aligned}
$$

$$
\begin{aligned}
& \text { and } \\
& \begin{aligned}
e^{z}= & { }_{0} F_{3}\left(\begin{array}{c|c}
- & 1 \\
1 / 4,1 / 2,3 / 4 & \frac{1}{256} z^{4}
\end{array}\right) \\
& +z_{0} F_{3}\left(\begin{array}{c|c|c}
- & 1 \\
1 / 2,3 / 4, & 5 / 4 & \frac{1}{256} z^{4}
\end{array}\right) \\
& +\frac{z^{2}}{2}{ }_{0} F_{3}\left(\begin{array}{c|c|c}
- & \frac{1}{256} z^{4}
\end{array}\right) \\
& +\frac{z^{3}}{6}{ }_{0} F_{3}\left(\begin{array}{ccc}
5 / 4,3 / 2 & - \\
5 / 4,3 / 2,7 / 4 & \frac{1}{256} z^{4}
\end{array}\right) .
\end{aligned}
\end{aligned}
$$

Note that the first representation corresponds to the decomposition of the exponential function in the even and odd parts. Moreover, the general case of the three above relations is given as

$e^{z}=\sum_{k=0}^{m-1} \frac{z^{k}}{k !}{ }_{1} F_{m}\left(\frac{1+k}{m}, \frac{2+k}{m}, \ldots, \frac{m+k}{m} \mid \frac{1}{m^{m}} z^{m}\right)$.

Example 2. Since ${ }_{1} F_{0}\left(\begin{array}{c}a \\ -\end{array} \mid z\right)=(1-z)^{-a}$, identity (2) for $m=2,3,4$, respectively, reads as

$$
\begin{aligned}
& (1-z)^{-a}={ }_{2} F_{1}\left(\begin{array}{cl}
a / 2, & (a+1) / 2 \\
1 / 2
\end{array} \mid z^{2}\right) \\
& +a z_{2} F_{1}\left(\begin{array}{c|c}
(a+1) / 2,(a+2) / 2 \\
3 / 2
\end{array} \mid z^{2}\right),
\end{aligned}
$$

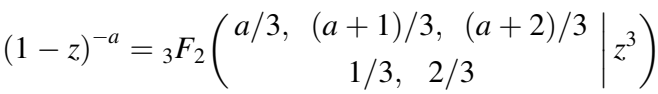

$$
\begin{aligned}
& +a \frac{z}{1 !}{ }_{3} F_{2}\left(\begin{array}{cc}
(a+1) / 3, & (a+2) / 3, \\
2 / 3, & 4 / 3
\end{array} \mid z^{3}\right) \\
& +a(a+1) \frac{z^{2}}{2}{ }_{3} F_{2}\left(\begin{array}{c}
(a+2) / 3, \\
4 / 3,5 / 3 \\
(a+3) / 3,(a+4) / 3
\end{array} \mid z^{3}\right),
\end{aligned}
$$

and

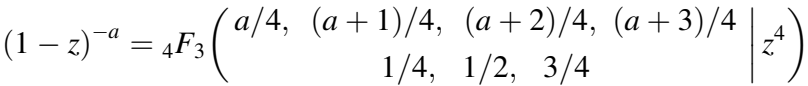

$$
\begin{aligned}
& +a z_{4} F_{3}\left(\begin{array}{c}
(a+1) / 4, \\
(a+2) / 4,(a+3) / 4, \\
1 / 2, \quad 3 / 4, \quad 5 / 4
\end{array} \mid z^{4}\right) \\
& +a(a+1) \frac{z^{2}}{2}{ }_{4} F_{3} \\
& \left(\begin{array}{c}
(a+2) / 4, \quad(a+3) / 4,(a+4) / 4,(a+5) / 4 \\
3 / 4, \quad 5 / 4, \quad 3 / 2
\end{array} \mid z^{4}\right) \\
& +a(a+1)(a+2) \frac{z^{3}}{6}{ }^{4} F_{3} \\
& \left(\begin{array}{c}
(a+3) / 4,(a+4) / 4,(a+5) / 4, \\
5 / 4, \quad 3 / 2, \quad 7 / 4
\end{array} \mid z^{4}\right) .
\end{aligned}
$$

Example 3. First, replacing $(p, q)=(0,1)$ in (2) for $m=$ 2 yields 


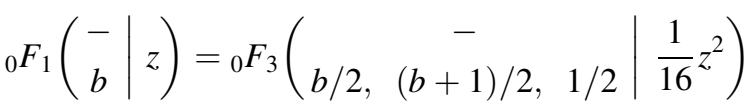

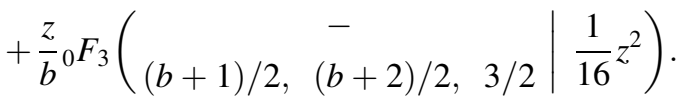

and

$$
\begin{aligned}
& \sin z=z_{0} F_{3}\left(\begin{array}{c|c}
- & 1 \\
1 / 2,3 / 4,5 / 4 & \frac{1}{256} z^{4}
\end{array}\right) \\
& -\frac{z^{3}}{6}{ }_{0} F_{3}\left(\begin{array}{c|c}
5 / 4,3 / 2,7 / 4 & \left.\frac{1}{256} z^{4}\right) .
\end{array}\right.
\end{aligned}
$$

Application 2. A generalization of Euler's identity.

If $m=2$ is replaced in (2), then we have

$$
\begin{aligned}
& { }_{p} F_{q}\left(\begin{array}{ccc}
a_{1}, a_{2}, \ldots & a_{p} \\
b_{1}, & b_{2}, \ldots & b_{q}
\end{array} \mid z\right)={ }_{2 p} F_{2 q+1}\left(\begin{array}{cc}
a_{1} / 2,\left(1+a_{1}\right) / 2, \ldots & a_{p} / 2,\left(1+a_{p}\right) / 2 \\
b_{1} / 2,\left(1+b_{1}\right) / 2, \ldots & b_{q} / 2,\left(1+b_{q}\right) / 2,1 / 2
\end{array} \mid 4^{p-q-1} z^{2}\right) \\
& +\frac{a_{1} a_{2} \ldots a_{p}}{b_{1} b_{2} \ldots b_{q}} z_{2 p} F_{2 q+1}\left(\begin{array}{ccc}
\left(a_{1}+1\right) / 2, & \left(a_{1}+2\right) / 2, \ldots & \left(a_{p}+1\right) / 2,\left(a_{p}+2\right) / 2 \\
\left(b_{1}+1\right) / 2, & \left(b_{1}+2\right) / 2, \ldots & \left(b_{q}+1\right) / 2,\left(b_{q}+2\right) / 2,3 / 2
\end{array} \mid 4^{p-q-1} z^{2}\right) .
\end{aligned}
$$

By noting that $z$ may be a complex variable, $z=i x$ in (4) gives

$$
\begin{array}{r}
{ }_{p} F_{q}\left(\begin{array}{ccc}
a_{1}, a_{2}, \ldots & a_{p} \\
b_{1}, b_{2}, \ldots & b_{q}
\end{array} \mid i x\right)={ }_{2 p} F_{2 q+1}\left(\begin{array}{cc}
a_{1} / 2,\left(1+a_{1}\right) / 2, \ldots & a_{p} / 2,\left(1+a_{p}\right) / 2 \\
b_{1} / 2,\left(1+b_{1}\right) / 2, \ldots & b_{q} / 2,\left(1+b_{q}\right) / 2,1 / 2
\end{array} \mid-4^{p-q-1} x^{2}\right) \\
+i \frac{a_{1} a_{2} \ldots a_{p}}{b_{1} b_{2} \ldots b_{q}} x_{2 p} F_{2 q+1}\left(\begin{array}{ccc}
\left(a_{1}+1\right) / 2,\left(a_{1}+2\right) / 2, \ldots & \left(a_{p}+1\right) / 2,\left(a_{p}+2\right) / 2 \\
\left(b_{1}+1\right) / 2,\left(b_{1}+2\right) / 2, \ldots & \left(b_{q}+1\right) / 2,\left(b_{q}+2\right) / 2,3 / 2
\end{array} \mid-4^{p-q-1} x^{2}\right)
\end{array}
$$

Now since the cosine and sine functions can be written as

$$
\begin{aligned}
& \cos z={ }_{0} F_{1}\left(\begin{array}{c|c}
- & -\frac{1}{4} z^{2}
\end{array}\right) \text { and } \sin z \\
& =z_{0} F_{1}\left(\begin{array}{c|c}
- & -\frac{1}{4} z^{2}
\end{array}\right) \text {, }
\end{aligned}
$$

relation (3), respectively, yields

$$
\begin{aligned}
& \cos z={ }_{0} F_{3}\left(\begin{array}{c|c}
- & - \\
1 / 4,1 / 2,3 / 4 & \frac{1}{256} z^{4}
\end{array}\right) \\
& -\frac{z^{2}}{2}{ }_{0} F_{3}\left(\begin{array}{c|c}
3 / 4,5 / 4,3 / 2 & \frac{1}{256} z^{4}
\end{array}\right) \text {, }
\end{aligned}
$$

which is a generalization of Euler's identity for $p=q=0$, because

$$
\begin{aligned}
{ }_{0} F_{0}(-\mid i x)= & { }_{0} F_{1}\left(\begin{array}{c}
- \\
1 / 2
\end{array} \mid-x^{2} / 4\right) \\
& +i x_{0} F_{1}\left(\begin{array}{c|c}
- & -x^{2} / 4 \\
3 / 2 &
\end{array}\right) \\
& \Leftrightarrow e^{i x}=\cos x+i \sin x .
\end{aligned}
$$

Subsequently, the well-known hyperbolic functions [2, 16] can be generalized via (4) and (5) and be defined, respectively, as

$$
\begin{array}{r}
{ }_{p} C h_{q}\left(\begin{array}{ccc}
a_{1}, a_{2}, \ldots & a_{p} \\
b_{1}, b_{2}, \ldots & b_{q}
\end{array} \mid x\right)=\frac{1}{2}\left({ }_{p} F_{q}\left(\begin{array}{cc}
a_{1}, a_{2}, \ldots & a_{p} \\
b_{1}, b_{2}, \ldots & b_{q}
\end{array} \mid x\right)+{ }_{p} F_{q}\left(\begin{array}{cc}
a_{1}, a_{2}, \ldots & a_{p} \\
b_{1}, b_{2}, \ldots & b_{q}
\end{array} \mid-x\right)\right) \\
\quad={ }_{2 p} F_{2 q+1}\left(\begin{array}{cc}
a_{1} / 2,\left(1+a_{1}\right) / 2, \ldots & a_{p} / 2,\left(1+a_{p}\right) / 2 \\
b_{1} / 2,\left(1+b_{1}\right) / 2, \ldots & b_{q} / 2,\left(1+b_{q}\right) / 2,1 / 2
\end{array} \mid 4^{p-q-1} x^{2}\right)
\end{array}
$$


Table 1 Characteristics of ten sequences of orthogonal polynomials

\begin{tabular}{|c|c|c|}
\hline Symbol & Weight function & Kind, interval and parameters constraint \\
\hline$P_{n}^{(u, v)}(x)$ & $W\left(\begin{array}{cc|c}-u-v, & -u+v \\
-1, & 0, & 1\end{array} \mid x\right)=(1-x)^{u}(1+x)^{v}$ & Infinite, $[-1,1], \forall n, u>-1, v>-1$ \\
\hline$L_{n}^{(u)}(x)$ & $W\left(\begin{array}{cc|c}-1, & u \\
0, & 1, & 0\end{array} \mid x\right)=x^{u} \exp (-x)$ & Infinite, $[0, \infty), \forall n, u>-1$ \\
\hline$H_{n}(x)$ & 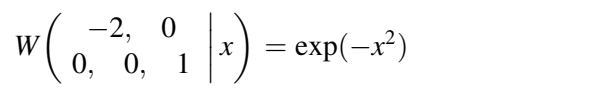 & Infinite, $(-\infty, \infty)$ \\
\hline$J_{n}^{(u, v)}(x)$ & $W\left(\begin{array}{cc|c}-2 u, & v \\
1, & 0, & 1\end{array}\right)=\left(1+x^{2}\right)^{-u} e^{v \arctan x}$ & Finite, $(-\infty, \infty), \max n<u-1 / 2, v \in \mathbb{R}$ \\
\hline$M_{n}^{(u, v)}(x)$ & $W\left(\begin{array}{cc|c}-u, & v \\
1, & 1, & 0\end{array}\right)=x^{v}(x+1)^{-(u+v)}$ & Finite, $[0, \infty), \max n<(u-1) / 2, v>-1$ \\
\hline$N_{n}^{(u)}(x)$ & 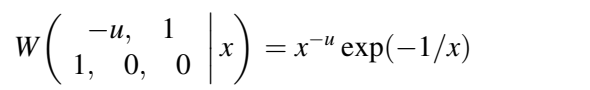 & Finite, $[0, \infty), \max n<(u-1) / 2$ \\
\hline$S_{n}\left(\begin{array}{cc|}-2 u-2 v-2, & 2 u \\
-1, & 1\end{array}\right)$ & 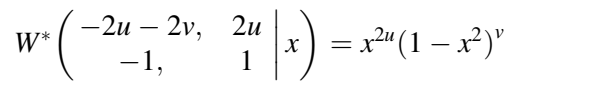 & Infinite, $[-1,1], u>-1 / 2, v>-1$ \\
\hline$S_{n}\left(\begin{array}{cc|c}-2, & 2 u & x \\
0, & 1 & x\end{array}\right)$ & $W^{*}\left(\begin{array}{cc|c}-2, & 2 u \\
0, & 1 & x\end{array}\right)=x^{2 u} \exp \left(-x^{2}\right)$ & Infinite, $(-\infty, \infty), u>-1 / 2$ \\
\hline$S_{n}\left(\begin{array}{cc}-2 u-2 v+2, & -2 u \\
1, & 1\end{array} \mid x\right.$ & $W^{*}\left(\begin{array}{cc|}-2 u-2 v, & -2 u \\
1, & 1\end{array} \mid x\right)=x^{-2 u}\left(1+x^{2}\right)^{-v}$ & Finite, $(-\infty, \infty), \max n<u+v-1 / 2, u<1 / 2, v>0$ \\
\hline$S_{n}\left(\begin{array}{cc|c}-2 u+2, & 2 & x \\
1, & 0 & \end{array}\right)$ & $W^{*}\left(\begin{array}{cc|c}-2 a, & 2 & x \\
1, & 0 & x\end{array}\right)=x^{-2 a} \exp \left(-1 / x^{2}\right)$ & Finite, $(-\infty, \infty), \max n<u-1 / 2$ \\
\hline
\end{tabular}

and

$$
\begin{aligned}
& { }_{p} S h_{q}\left(\begin{array}{cc}
a_{1}, a_{2}, \ldots & a_{p} \\
b_{1}, b_{2}, \ldots & b_{q}
\end{array} \mid x\right)=\frac{1}{2}\left({ }_{p} F_{q}\left(\begin{array}{ccc}
a_{1}, a_{2}, \ldots & a_{p} \\
b_{1}, b_{2}, \ldots & b_{q}
\end{array} \mid x\right)-{ }_{p} F_{q}\left(\begin{array}{cc}
a_{1}, a_{2}, \ldots & a_{p} \\
b_{1}, b_{2}, \ldots & b_{q}
\end{array} \mid-x\right)\right) \\
= & \frac{a_{1} a_{2} \ldots a_{p}}{b_{1} b_{2} \ldots b_{q}} x_{2 p} F_{2 q+1}\left(\begin{array}{cc}
\left(a_{1}+1\right) / 2,\left(a_{1}+2\right) / 2, \ldots & \left(a_{p}+1\right) / 2,\left(a_{p}+2\right) / 2 \\
\left(b_{1}+1\right) / 2,\left(b_{1}+2\right) / 2, \ldots & \left(b_{p}+1\right) / 2,\left(b_{p}+1\right) / 2,3 / 2
\end{array} \mid 4^{p-q-1} x^{2}\right) .
\end{aligned}
$$

It is clear that for $(p, q)=(0,0)$, relations (6) and (7) reduce to

$$
\begin{aligned}
{ }_{0} C h_{0}\left(\begin{array}{l}
-\mid x) \\
-\mid
\end{array}\right) & \cosh x=\frac{1}{2}\left(e^{x}+e^{-x}\right) \text { and }{ }_{0} \operatorname{Sh}_{0}\left(\begin{array}{c}
-\mid x) \\
-\mid x
\end{array}\right. \\
& =\sinh x=\frac{1}{2}\left(e^{x}-e^{-x}\right) .
\end{aligned}
$$

Application 3. A decomposition formula for classical hypergeometric orthogonal polynomials: there are ten sequences of hypergeometric polynomials [7, 12-14] that are orthogonal with respect to the Pearson distribution family $W\left(\begin{array}{c}d, e \\ a, b, c\end{array} \mid x\right)=\exp \left(\int \frac{d x+e}{a x^{2}+b x+c} d x\right) \quad(a, b, c, d, e \in \mathbb{R})$,

and its symmetric analog [11]

$W^{*}\left(\begin{array}{cc}r, & s \\ p, & q\end{array} \mid x\right)=\exp \left(\int \frac{r x^{2}+s}{x\left(p x^{2}+q\right)} d x\right) \quad(p, q, r, s \in \mathbb{R})$.
Five of them are infinitely orthogonal with respect to special cases of the two above-mentioned weight functions and five other ones are finitely orthogonal [12-14] which are limited to some parametric constraints. The following Table 1 shows their main characteristics. where the sequence

$$
\begin{gathered}
\Phi_{n}(x)=S_{n}\left(\begin{array}{cc|c}
r, & s \\
p, & q & x
\end{array}\right)=\sum_{k=0}^{[n / 2]}\left(\begin{array}{c}
{[n / 2]} \\
k
\end{array}\right) \\
\left(\prod_{i=0}^{[n / 2]-(k+1)} \frac{\left(2 i+(-1)^{n+1}+2[n / 2]\right) p+r}{\left(2 i+(-1)^{n+1}+2\right) q+s}\right) x^{n-2 k}
\end{gathered}
$$

is a basic class of symmetric orthogonal polynomials [12] satisfying the equation

$$
\begin{aligned}
& x^{2}\left(p x^{2}+q\right) \Phi_{n}^{\prime \prime}(x)+x\left(r x^{2}+s\right) \Phi_{n}^{\prime}(x)-\left(n(r+(n-1) p) x^{2}\right. \\
& \left.\quad+\left(1-(-1)^{n}\right) s / 2\right) \Phi_{n}(x)=0 .
\end{aligned}
$$


For example, consider the shifted Jacobi polynomials [20]

$$
\begin{gathered}
P_{n,+}^{(\alpha, \beta)}(x)={ }_{2} F_{1}\left(\begin{array}{c|c}
-n, & n+\alpha+\beta+1 \\
\alpha+1
\end{array} \mid x\right) \\
(\alpha, \beta>-1),
\end{gathered}
$$

which are orthogonal with respect to the shifted beta distribution on $[0,1]$ as

$$
\begin{aligned}
& \int_{0}^{1} x^{\alpha}(1-x)^{\beta} P_{n,+}^{(\alpha, \beta)}(x) P_{m,+}^{(\alpha, \beta)}(x) d x \\
& \quad=\frac{n ! \Gamma^{2}(\alpha+1) \Gamma(n+\beta+1)}{(2 n+\alpha+\beta+1) \Gamma(n+\alpha+\beta+1) \Gamma(n+\alpha+1)} \delta_{n, m} .
\end{aligned}
$$

Another classical example are the Laguerre polynomials

$$
y=\widehat{L}_{n}^{(\alpha)}(x)={ }_{1} F_{1}\left(\begin{array}{c}
-n \\
\alpha+1
\end{array} \mid x\right) \quad(\alpha>-1),
$$

that satisfy the differential equation [7]

$x y^{\prime \prime}+(\alpha+1-x) y^{\prime}+n y=0$,

and the orthogonality relation [7]

$\int_{0}^{\infty} x^{\alpha} e^{-x} \widehat{L}_{n}^{(\alpha)}(x) \widehat{L}_{m}^{(\alpha)}(x) \mathrm{d} x=\frac{n ! \Gamma(\alpha+1)}{(\alpha+1)_{n}} \delta_{n, m}$.

If (8) is replaced in (2) for, e.g., $m=2$, then one gets (see also [10] in this regard)

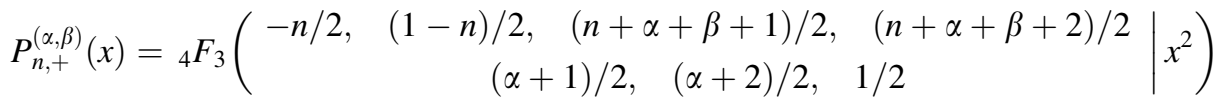

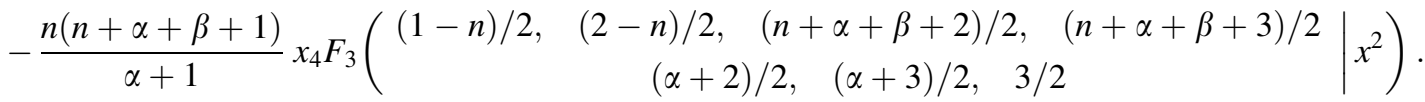

Hence, two sequences of polynomials can be defined by (9)

as

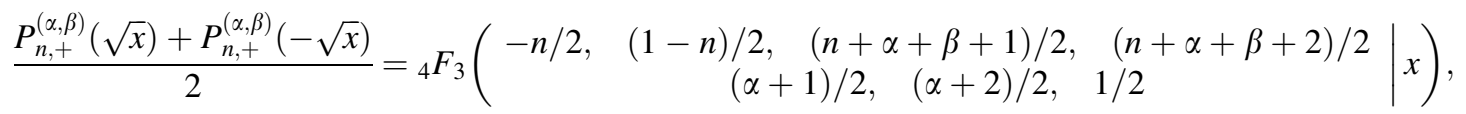

and

$$
\begin{aligned}
& \frac{P_{n,+}^{(\alpha, \beta)}(\sqrt{x})-P_{n,+}^{(\alpha, \beta)}(-\sqrt{x})}{2 \sqrt{x}}=-\frac{n(n+\alpha+\beta+1)}{\alpha+1} \\
& \times{ }_{4} F_{3}\left(\begin{array}{cc}
(1-n) / 2, & \begin{array}{c}
(2-n) / 2, \\
(n+\alpha+\beta+2) / 2,
\end{array}(n+\alpha+\beta+3) / 2 \\
(\alpha+2) / 2, \quad(\alpha+3) / 2, \quad 3 / 2
\end{array} \mid x\right) .
\end{aligned}
$$


If (10) is replaced in (2) for e.g. $m=3$, then one gets

$$
\begin{aligned}
\widehat{L}_{n}^{(\alpha)}(x)= & { }_{3} F_{5}\left(\begin{array}{c}
-n / 3,(1-n) / 3, \quad(2-n) / 3 \\
(\alpha+1) / 3,(\alpha+2) / 3, \quad(\alpha+3) / 3,1 / 3,2 / 3
\end{array} \mid \frac{x^{3}}{27}\right) \\
& -\frac{n}{\alpha+1} x_{3} F_{5} \\
& \left(\begin{array}{c}
(1-n) / 3,(2-n) / 3, \quad(3-n) / 3 \\
(\alpha+2) / 3,(\alpha+3) / 3,(\alpha+4) / 3,2 / 3,4 / 3
\end{array}\right. \\
& +\frac{n(n-1)}{2(\alpha+1)(\alpha+2)} x_{3}^{2} F_{5} \\
& \left(\begin{array}{c}
(2-n) / 3,(3-n) / 3,(4-n) / 3 \\
(\alpha+3) / 3,(\alpha+4) / 3,(\alpha+5) / 3,4 / 3,5 / 3
\end{array} \mid \frac{x^{3}}{27}\right) .
\end{aligned}
$$

See also [9] in the sense of incomplete symmetric orthogonal polynomials of Laguerre type. Some other works related to classical hypergeometric orthogonal polynomials can be found in [3, 4, 19].

Application 4. The classical summation theorems of hypergeometric series (such as the Gauss, Kummer and Bailey theorems for ${ }_{2} F_{1}$ and the Watson and Dixon theorems for ${ }_{3} F_{2}$ ) play an important role in evaluating hypergeometric series at specific points [18]. In this section, by expressing the aforesaid theorems we employ identity (2) for them.

- Gauss's theorem:

${ }_{2} F_{1}\left(\begin{array}{cc|c}a, & b & 1 \\ c & 1\end{array}\right)=\frac{\Gamma(c) \Gamma(c-a-b)}{\Gamma(c-a) \Gamma(c-b)}$,

provided that $\operatorname{Re}(c-a-b)>0$.

- Gauss's second theorem:

${ }_{2} F_{1}\left(\begin{array}{c|c}a, \quad b & 1 \\ (a+b+1) / 2 & \frac{1}{2}\end{array}\right)=\frac{\sqrt{\pi} \Gamma\left(\frac{a+b+1}{2}\right)}{\Gamma\left(\frac{a+1}{2}\right) \Gamma\left(\frac{b+1}{2}\right)}$,

provided that $\operatorname{Re}(a)>-1$ and $\operatorname{Re}(b)>-1$.

- Kummer's theorem:

${ }_{2} F_{1}\left(\begin{array}{c}a, b \\ 1+a-b\end{array} \mid-1\right)=\frac{\Gamma(1+a-b) \Gamma\left(1+\frac{a}{2}\right)}{\Gamma(1+a) \Gamma\left(1+\frac{a}{2}-b\right)}$. provided that $\operatorname{Re}(a)>-1, \operatorname{Re}(b)<1$ and $\operatorname{Re}(a-b)$ $>-1$.

- Bailey's theorem [18]:

${ }_{2} F_{1}\left(\begin{array}{ll|l}a, & 1-a & \frac{1}{2}\end{array}\right)=\frac{\Gamma\left(\frac{c}{2}\right) \Gamma\left(\frac{c+1}{2}\right)}{\Gamma\left(\frac{c+a}{2}\right) \Gamma\left(\frac{c-a+1}{2}\right)}$.

provided that $\operatorname{Re}(c)>\operatorname{Re}(a)>0$.

- Watson's theorem [18]:

$$
\begin{aligned}
{ }_{3} F_{2} & \left(\begin{array}{cc|c}
a, \quad b, & c & 1 \\
(a+b+1) / 2, & 2 c
\end{array}\right) \\
= & \frac{\sqrt{\pi} \Gamma\left(c+\frac{1}{2}\right) \Gamma\left(\frac{a+b+1}{2}\right) \Gamma\left(c-\frac{a+b-1}{2}\right)}{\Gamma\left(\frac{a+1}{2}\right) \Gamma\left(\frac{b+1}{2}\right) \Gamma\left(c-\frac{a-1}{2}\right) \Gamma\left(c-\frac{b-1}{2}\right)} .
\end{aligned}
$$

provided that $\operatorname{Re}(2 c-a-b)>-1$.

- Dixon's theorem [18]:

$$
\begin{aligned}
{ }_{3} F_{2} & \left(\begin{array}{c}
a, \quad b, \quad c \\
1+a-b, 1+a-c
\end{array} \mid 1\right) \\
= & \frac{\Gamma\left(1+\frac{a}{2}\right) \Gamma(1+a-b) \Gamma(1+a-c) \Gamma\left(1+\frac{a}{2}-b-c\right)}{\Gamma(1+a) \Gamma\left(1+\frac{a}{2}-b\right) \Gamma\left(1+\frac{a}{2}-c\right) \Gamma(1+a-b-c)} .
\end{aligned}
$$

provided that $\operatorname{Re}(a-2 b-2 c)>-2$.

Now, if we substitute Gauss's theorem into (8) for e.g. $m=2,3$, then we, respectively, obtain

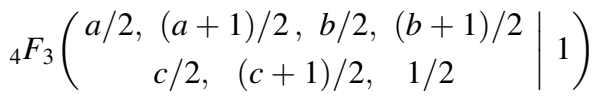

$$
\begin{aligned}
& +\frac{a b}{c}{ }_{4} F_{3}\left(\begin{array}{c|c}
(a+1) / 2,(a+2) / 2,(b+1) / 2,(b+2) / 2 & 1 \\
(c+1) / 2, & (c+2) / 2,3 / 2
\end{array}\right) \\
& =\frac{\Gamma(c) \Gamma(c-a-b)}{\Gamma(c-a) \Gamma(c-b)},
\end{aligned}
$$

and

$$
\begin{aligned}
& { }_{6} F_{5}\left(\begin{array}{c|c}
a / 3,(a+1) / 3,(a+2) / 3, b / 3,(b+1) / 3,(b+2) / 3 & 1 \\
c / 3,(c+1) / 3,(c+2) / 3,1 / 3,2 / 3 &
\end{array}\right)
\end{aligned}
$$

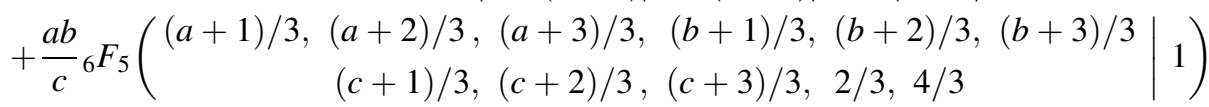

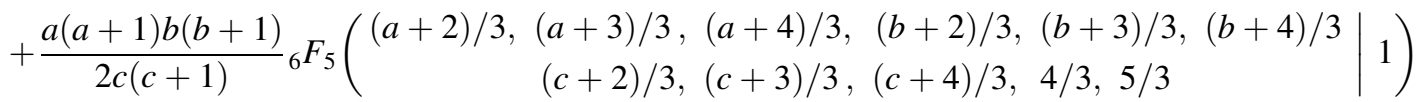

$$
\begin{aligned}
& =\frac{\Gamma(c) \Gamma(c-a-b)}{\Gamma(c-a) \Gamma(c-b)} .
\end{aligned}
$$


The general case of the two above identities for any natural $m$ is as

$\sum_{k=0}^{m-1} \frac{(a)_{k}(b)_{k}}{(c)_{k} k !}{ }_{2 m+1} F_{2 m}\left(\begin{array}{c}\frac{a+k}{m}, \ldots, \frac{a+m-1+k}{m}, \frac{b+k}{m}, \ldots, \frac{b+m-1+k}{m}, 1 \\ \frac{c+k}{m}, \ldots, \frac{c+m-1+k}{m}, \frac{1+k}{m}, \ldots, \frac{m+k}{m}\end{array} \mid 1\right)=\frac{\Gamma(c) \Gamma(c-a-b)}{\Gamma(c-a) \Gamma(c-b)}$.

And the general case for Gauss's second theorem takes the form

$$
\begin{aligned}
& \sum_{k=0}^{m-1} \frac{(a)_{k}(b)_{k} 2^{-k}}{\left(\frac{a+b+1}{2}\right)_{k} k !}{ }^{2 m+1} F_{2 m}\left(\begin{array}{c}
\frac{a+k}{m}, \ldots, \frac{a+m-1+k}{m}, \frac{b+k}{m}, \ldots, \frac{b+m-1+k}{m}, 1 \\
\frac{k+(a+b+1) / 2}{m}, \ldots, \frac{m-1+k+(a+b+1) / 2}{m}, \frac{1+k}{m}, \ldots, \frac{m+k}{m}
\end{array}\right) \\
& \quad=\frac{\sqrt{\pi} \Gamma\left(\frac{a+b+1}{2}\right)}{\Gamma\left(\frac{a+1}{2}\right) \Gamma\left(\frac{b+1}{2}\right)},
\end{aligned}
$$

and for Kummer theorem takes the form

$$
\begin{aligned}
& \left.\sum_{k=0}^{m-1} \frac{(a)_{k}(b)_{k}(-1)^{k}}{(1+a-b)_{k} k !} 2 m+1 F_{2 m}\left(\begin{array}{c}
\frac{a+k}{m}, \ldots, \frac{a+m-1+k}{m}, \frac{b+k}{m}, \ldots, \frac{b+m-1+k}{m}, 1 \\
\frac{1+a-b+k}{m}, \ldots, \frac{a-b+m+k}{m}, \frac{1+k}{m}, \ldots, \frac{m+k}{m}
\end{array}\right)(-1)^{m}\right) \\
& \quad=\frac{\Gamma(1+a-b) \Gamma\left(1+\frac{a}{2}\right)}{\Gamma(1+a) \Gamma\left(1+\frac{a}{2}-b\right)}
\end{aligned}
$$

and for Bailey theorem takes the form

$$
\begin{aligned}
& \sum_{k=0}^{m-1} \frac{(a)_{k}(1-a)_{k}(2)^{-k}}{(c)_{k} k !} 2 m+1 F_{2 m}\left(\begin{array}{r}
\frac{a+k}{m}, \ldots, \frac{a+m-1+k}{m}, \frac{1-a+k}{m}, \ldots, \frac{-a+m+k}{m}, 1 \\
\frac{c+k}{m}, \ldots, \frac{c+m-1+k}{m}, \frac{1+k}{m}, \ldots, \frac{m+k}{m}
\end{array}\right) 2^{-m} \\
& \quad=\frac{\Gamma\left(\frac{c}{2}\right) \Gamma\left(\frac{c+1}{2}\right)}{\Gamma\left(\frac{c+a}{2}\right) \Gamma\left(\frac{c-a+1}{2}\right)}
\end{aligned}
$$


and for Watson theorem takes the form

$$
\begin{aligned}
& \sum_{k=0}^{m-1} \frac{(a)_{k}(b)_{k}(c)_{k}}{((a+b+1) / 2)_{k}(2 c)_{k} k !} \times \\
& \left.\quad \begin{array}{c}
\frac{a+k}{m}, \ldots, \frac{a+m-1+k}{m}, \frac{b+k}{m}, \ldots, \frac{b+m-1+k}{m}, \frac{c+k}{m}, \ldots, \frac{c+m-1+k}{m}, 1 \\
\left.\frac{k+(a+b+1) / 2}{m}, \ldots, \frac{m-1+k+(a+b+1) / 2}{m}, \frac{2 c+k}{m}, \ldots, \frac{2 c+m-1+k}{m}, \frac{1+k}{m}, \ldots, \frac{m+k}{m} \mid 1\right)
\end{array}\right) \\
& \quad=\frac{\sqrt{\pi} \Gamma\left(c+\frac{1}{2}\right) \Gamma\left(\frac{a+b+1}{2}\right) \Gamma\left(c-\frac{a+b-1}{2}\right)}{\Gamma\left(\frac{a+1}{2}\right) \Gamma\left(\frac{b+1}{2}\right) \Gamma\left(c-\frac{a-1}{2}\right) \Gamma\left(c-\frac{b-1}{2}\right)},
\end{aligned}
$$

and finally for Dixon's theorem takes the form

$$
\begin{aligned}
& \sum_{k=0}^{m-1} \frac{(a)_{k}(b)_{k}(c)_{k}}{(1+a-b)_{k}(1+a-c)_{k} k !} \times \\
& 3_{3 m+1} F_{3 m}\left(\begin{array}{c}
\frac{a+k}{m}, \ldots, \frac{a+m-1+k}{m}, \frac{b+k}{m}, \ldots, \frac{b+m-1+k}{m}, \frac{c+k}{m}, \ldots, \frac{c+m-1+k}{m}, 1 \\
\frac{1+a-b+k}{m}, \ldots, \frac{a-b+m+k}{m}, \frac{1+a-c+k}{m}, \ldots, \frac{a-c+m+k}{m}, \frac{1+k}{m}, \ldots, \frac{m+k}{m}
\end{array} \mid \begin{array}{l}
1 \\
1
\end{array}\right) \\
& =\frac{\Gamma\left(1+\frac{a}{2}\right) \Gamma(1+a-b) \Gamma(1+a-c) \Gamma\left(1+\frac{a}{2}-b-c\right)}{\Gamma(1+a) \Gamma\left(1+\frac{a}{2}-b\right) \Gamma\left(1+\frac{a}{2}-c\right) \Gamma(1+a-b-c)} .
\end{aligned}
$$

Acknowledgments This work is supported by a Grant from "Iran National Science Foundation” No. 92026373.

Open Access This article is distributed under the terms of the Creative Commons Attribution 4.0 International License (http://crea tivecommons.org/licenses/by/4.0/), which permits unrestricted use, distribution, and reproduction in any medium, provided you give appropriate credit to the original author(s) and the source, provide a link to the Creative Commons license, and indicate if changes were made.

\section{References}

1. Ancarani, L.U., Gasaneo, G.: Derivatives of any order of the hypergeometric function ${ }_{p} F_{q}\left(a_{1}, \ldots, a_{p} ; b_{1}, \ldots, b_{q} ; z\right)$ with respect to the parameters ai and bi. J. Phys. A: Math. Theor. 43, 085210 (2010)

2. Andrews, G.E., Askey, R., Roy, R.: Special Functions. Cambridge University Press, Cambridge (2000)

3. Cheikh, Y.B.: Decomposition of Laguerre polynomials with respect to the cyclic group of order n. Appl. Math. Inform. 81, 51-64 (1997)

4. Cheikh, Y.B.: Decomposition of the Bessel functions with respect to the cycle group of order $n$. Matematiche (Catania) 52, 365-378 (1997)
5. Chaudhry, M.A., Qadir, A., Srivastava, H.M., Paris, R.B.: Extended hypergeometric and confluent hypergeometric functions. Appl. Math. Comput. 159, 589-602 (2004)

6. Gradshteyn, I.S., Ryzhik, I.M.: Table of Integrals, Series, and Products, 7th edn. Academic Press, Elsevier Inc (2007)

7. Koekoek, R., Lesky, P.A., Swarttouw, R.F.: Hypergeometric Orthogonal Polynomials and Their q-Analogues, Springer Monographs in Mathematics. Springer, Heidelberg (2010)

8. Koepf, W.: Power series in computer algebra. J. Symb. Comput. 11, 581-603 (1992)

9. Masjed-Jamei, M., Koepf, W.: On incomplete symmetric orthogonal polynomials of Laguerre type. Appl. Anal. 90, 769-775 (2011)

10. Masjed-Jamei, M., Koepf, W.: On incomplete symmetric orthogonal polynomials of Jacobi type. Integral Transforms Spec. Funct. 21, 655-662 (2010)

11. Masjed-Jamei, M.: On relationships between classical Pearson distributions and Gauss hypergeometric function. Acta Applicandae Mathematicae 109, 401-411 (2010)

12. Masjed-Jamei, M.: A basic class of symmetric orthogonal polynomials using the extended Sturm-Liouville theorem for symmetric functions. J. Math. Anal. Appl. 325, 753-775 (2007)

13. Masjed-Jamei, M.: Classical orthogonal polynomials with weight function: $\left((a x+b)^{2}+(c x+d)^{2}\right)^{-p} \exp (q \arctan a x+b /$ $c x+d)$ on $(-\infty, \infty)$ and a generalization of $\mathrm{T}$ and $\mathrm{F}$ distributions. Integral Transforms Spec. Funct. 15, 137-153 (2004) 
14. Masjed-Jamei, M.: Three finite classes of hypergeometric orthogonal polynomials and their application in functions approximation. Integral Transforms Spec. Funct. 13, 169-190 (2002)

15. Mathai, A.M., Saxena, R.K.: Generalized Hypergeometric Functions with Applications in Statistics and Physical Sciences, Lecture Notes in Mathematics, vol. 348. Springer, Berlin, Heidelberg, New York (1973)

16. Nikiforov, A.F., Uvarov, V.B.: Special Functions of Mathematical Physics. Birkhauser, Boston (1988)
17. Ozergin, E., Ozarslan, M.A., Altin, A.: Extension of gamma, beta and hypergeometric functions. J. Comput. Appl. Math. 235, 4601-4610 (2011)

18. Prudnikov, A.P., Brychkov, Y.A., Marichev, O.I.: Integrals and Series, Volume 3: More Special Functions. Gordon and Breach Science Publishers, Philadelphia (1990)

19. Ronveaux, A., Zarzo, A., Area, I., Godoy, E.: Decomposition of polynomials with respect to the cyclic group of order m. J. Symb. Comput. 28, 755-765 (1999)

20. Szegö, G.: Orthogonal Polynomials. AMS, Providence (1978) 